linked polystyrene in bead form, 15-30 mesh/in. Its water regain at saturation was $0.75 \mathrm{gm}$. water per gm. dry $\mathrm{H}^{+}$resin, which corresponds to a nominal divinylbenzene content of 10-12 per cent ${ }^{2}$.

The work described in this and the preceding communication was carried out as part of the programme of the Food Investigation Organization of the Department of Scientific and Industrial Research.

Low Temperature Station

S. M. PARTRIDGE

for Research in Biochemistry and Biophysies, University of Cambridge, and

Department of Scientific and Industrial Research. Oct. 30.

' Pepper, K. W., J. App. Chem., 1, 124 (1951).

3 Richardson, R. W., Nature, 164, 916 (1949).

${ }^{3}$ Richardson, R. W., J. Chem. Soc., 910 (1951).

- Kunin, R., Anal. Chem., 21, 87 (1949).

- Deuel, H., Solms, J., and Anyas-Weisz, L., Helv. Chim. Acta, 33, $2171(1950)$.

\section{Determination of Sodium Oleate in Dilute Aqueous Solution}

THERE is no simple, satisfactory method for determining small amounts $\left(<10^{-3} M\right)$ of carboxylic soaps in aqueous solution. On the other hand, there are several methods of estimating synthetic ionic detergents, such as long-chain alkyl sulphates and quaternary ammonium salts. Of particular interest is the method of Epton ${ }^{1}$, in which the titration of anionic against cationic detergent is carried out using a partition end-point with methylene blue as indicator. We have now found that sodium oleate can be estimated by a modification of this method, if certain precautions are taken. Considerations of solubility led us to choose sodium oleate to illustrate the method, although it is clearly applicable to other soaps of similar constitution.

A dilution series of aqueous sodium oleate was prepared in the range $1.0 \times 10^{-3} \mathrm{M}$ down to $1.0 \times$ $10^{-4} M$. The solutions were maintained at $p \mathrm{H} 10$ by the presence of an excess of sadium hydroxide. Should the $p \mathrm{H}$ fall appreciably below this, the presence of 'acid soap' invalidates the method. $10.0 \mathrm{ml}$. of $5.0 \times 10^{-4} M$ cetyl trimethyl ammonium bromide was run into a $50-\mathrm{ml}$. stoppered cylinder containing $10 \mathrm{ml}$, chloroform. The oleate solution was then added from a burette, with frequent shaking. As the end-point was approached, the stability of the emulsion increased. Near the endpoint, $0.2 \mathrm{ml}$. of aqueous methylene blue solution ( 0.05 per cent) was added. The true end-point was obtained when the intensity of the dye colour in the chloroform phase became equal to that in the aqueous phase, as viewed against a background of diffuse light. The methylene blue in the chloroform phase becomes pink within about one minute after its addition, due to the presence of the alkali in the aqueous phase. Since the development of this pink colour tends to mask the end-point, the addition of the indicator must be delayed as long as possible. Accurate and reproducible results could only be obtained by delaying the addition of indicator until a marked increase in emulsion stability was observed; at this stage, the titration was about 95 per cent complete. The accompanying graph illustrates the precision of the method (circles).

As an independent means of estimating the oleate concentrations, we have developed a method based on the Langmuir surface balance. Using a micrometer-syringe, a known volume of the soap solution

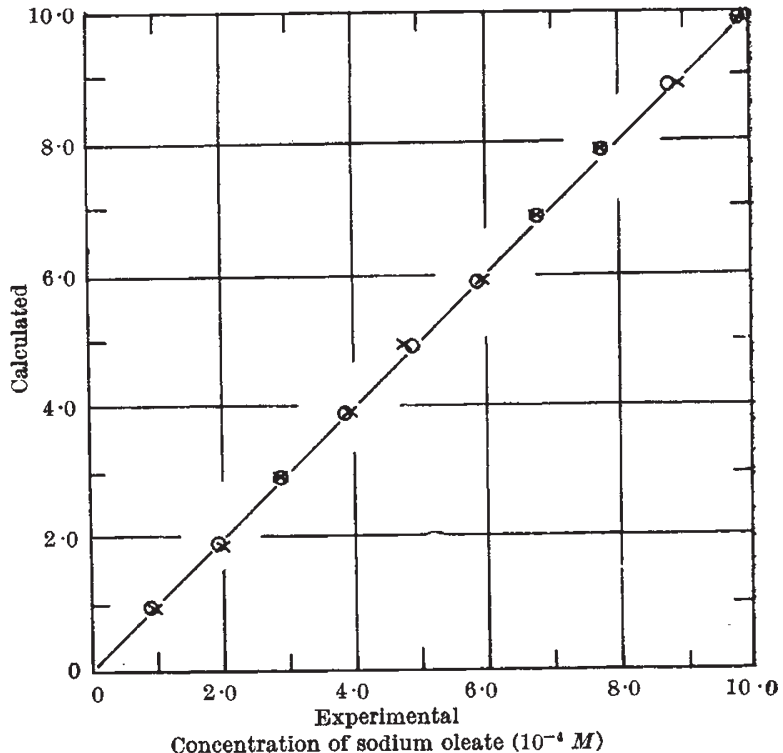

Comparison of titration methods for sodium oleate determinations : $O$, by partition titration ; $x$, by surface balance technique The straight line represents the th

was spread on a continuous recording surfacebalance ${ }^{2}$ containing, as substrate, a 25 per cent ammonium sulphate solution which was $0.01 \mathrm{~N}$ with respect to sulphuric acid. By measuring the collapse area of the derived oleic acid monolayer, it was possible to determine the oleate solution concentration. Results are shown in the graph (crosses).

Both methods are accurate to \pm 1 per cent. From the graph, it can be seen that they give the correct, calculated end-point over the whole of the investigated range.

Since the cetyl trimethyl ammonium bromide/ oleate titration method is based, fundamentally, on the formation of a complex between the cationic and anionic long-chain radicals, the presence of any undissociated fatty acid will interfere with the observed end-point. It is clear, therefore, that the sodium oleate solution must be sufficiently alkaline to ensure virtually complete ionization of the fatty acid. Thus, Epton (loc. cit.) has remarked, and we have confirmed, that neutral $(p H$ 8) solutions of oleate cannot be estimated by the partition method. We have found that a $p \mathrm{H}$ of (at least) 10 is necessary for complete fatty acid ionization and, hence, for obtaining the correct end-point. It is also of interest, in connexion with the surface-balance method, that unless the $p H$ of the spreading solution is (at least) 10 , complete spreading of the monolayer does not occur.

As might be expected, the partition titration method is affected by calcium ions $(0.01 M)$ present in the soap solution, due to the formation of un-ionized calcium oleate. Similarly, copper ions $(0.005 M)$ destroy the end-point by precipitating the basic copper oleate.

The cetyl trimethyl ammonium bromide solution was standardized by partition titration against pure sodium dodecyl sulphate.

Colloid Science Department,

J. GLazker

T. D. SMITH

University, Cambridge. Nov. 10

${ }^{1}$ Epton, Trans. Farad. Soc., 44, 226 (1948).

2 Glazer and Alexander, Trans. Farad. Soc., 47, 401 (1951). 